www.jmscr.igmpublication.org

Index Copernicus Value: 79.54

ISSN (e)-2347-176x ISSN (p) 2455-0450

crossrefDOI: https://dx.doi.org/10.18535/jmscr/v7i2.177

\title{
Peri-Operative Challenges in Patients with Complete Heart Block with Varied Cardiac Pacemakers and Implantable Cardiac Defibrillators
}

\author{
Authors \\ S R Karthick ${ }^{1}$, Satish Logidasan ${ }^{2}$, Kanimozhi $^{3}{ }^{3}$, Arulraj G.P ${ }^{4}$, Karthik.A ${ }^{5}$ \\ S Prasana Vathanan ${ }^{6}$, Ambal $^{7}$, C Sathish $^{8}$ \\ Corresponding Author \\ A Gowri Shankar \\ Department of Anesthesiology, Govt Stanley Medical College, TN- 600001, India \\ Email:agsmbbs@gmail.com
}

\begin{abstract}
The indications for CIEDs (Cardiac pacemakers and implantable cardiac defibrillators) in the general population is increasing worldwide. Patients with CIEDs presenting for a non cardiac surgery is on the rise. It is the Anaesthesiologist's duty to be well versed and take adequate perioperative care of the patients with CIEDs presenting for non cardiac surgeries. Here we present a case of 67 year old female with Carcinoma stomach with PPI insitu posted for subtotal gastrectomy.
\end{abstract}

\section{Introduction}

Artificial pacemakers are generally indicated for the treatment of symptomatic bradycardia of any origin $^{(1)}$. The spectrum of indications for permanent pacing is on a steady rise along with the technological advancements with respect to pacemakers rises parallely. Some of the specific indications include sick sinus syndrome, chronic bifascicular block, long QT syndrome etc.. ${ }^{(2)}$. With respect to internal cardiac defibrillators (ICDs) the latest advancement has significantly reduced the complications and widened the spectrum of indications also. When a patient with CIEDs presents for a non cardiac surgery, the intraoperative environment provides several opportunities to disturb proper functioning of the device. In addition the other comorbidities of the patient serves as an additional challenge for the Anaesthesiologist.

\section{Case Report}

A 67 year old female weighing $60 \mathrm{~kg}$ presented to SGE OP department with pain abdomen associated with vomiting for past 3 months. UGI scopy revealed an ulceroproliferative growth in fundus of stomach for which subtotal gastrectomy was planned after biopsy confirmation as malignancy. The patient had a permanent pacemaker placed 10 years ago for symptomatic bradycardia with complete heart block.

On the day before surgery pacemaker technician from the concerned company (Medtronics) was called to evaluate the working condition of the pacemaker. There was no intrinsic rhythm checked by applying magnet. Rate responsiveness was deactivated by applying programme. Preoperative night patient was premedicated with tablet Ranitidine 150mg and tablet Diazepam $5 \mathrm{mg}$. 
On the day of surgery, a cardiologist was kept available in the operating room. The company person was made to stay the whole day during surgery. Defibrillator with facilities for external temporary pacemaker was kept ready in the OR. Antiarrhythmics, vasopressors and other emergency drugs were kept ready. Surgeon was advised to use bipolar cautery instead of monopolar cautery to minimize electromagnetic interference.

The procedure was planned under General Anaesthesia. The pacemaker was originally in VVI mode at a fixed heart rate of 60 beats per minute. The mode was changed to VOO mode with the help of technical person. Later patient was preoxygenated with $100 \% \mathrm{O} 2$ for 3 minutes and monitors connected. Inj glycopyrrolate $0.2 \mathrm{mg}$ and Inj fentanyl 100mics was given intravenously. Patient was induced with Inj Propofol $100 \mathrm{mg}$ and Inj Veccuronium 6mg intravenously. Patient was intubated with $7.5 \mathrm{~mm}$ (ID) cuffed endotracheal tube. Anaesthesia was maintained with $0.2-2 \%$ sevoflurane with a 50:50 mixture of $\mathrm{O} 2: \mathrm{N} 2 \mathrm{O}$ and Inj morphine $10 \mathrm{mg}$ intravenous bolus. Intraoperatively pacemaker function was monitored by ECG. The artifact filter in the ECG monitor was disabled in order to detect pacing spikes. The ECG monitor was placed in diagnostic mode for accurate monitoring of QRS morphology and ST segment analysis. Under ultrasound guidance right internal jugularvein was cannulated with 7 Fr trilumen CVP catheter. After performing an Allen's test right radial artery was cannulated and beat to beat invasive BP was also monitored. An episode of hypotension with a BP of $80 / 60 \mathrm{mmHg}$ was managed with ephedrine bolus. After completion of procedure patient was reversed with $2.5 \mathrm{mg}$ Neostigmine and $0.4 \mathrm{mg}$ glycopyrolate intravenously and was extubated. The pacemaker mode was changed to original VVI mode and the patient was shifted to postoperative ICU for observation.

\section{Discussion}

\section{Pacemaker}

A pacemaker consists of an impulse generator and one or multiple leads to carry the electrical impulse to the patient's heart. Leads can be unipolar/ bipolar/ multipolar connected to the heart chambers through vena cava or on surface of heart epicardially ${ }^{(3)}$. The fundamental information required to understand normal pacemaker behaviour is the pacemaker code. The currently used North American Pacing and Electrophysiology/British Pacing and Electrophysiology Group (NASPE/BPEG) pacemaker codes are used to describe pacemaker types and function ${ }^{(2)}$

\section{Generic Codes for Pacemakers}

\begin{tabular}{|c|c|c|c|c|}
\hline $\begin{array}{c}\text { LETTER } \\
\mathbf{1} \\
\text { Chamber } \\
\text { paced }\end{array}$ & $\begin{array}{c}\text { LETTER } \\
\mathbf{2} \\
\text { Chamber } \\
\text { sensed }\end{array}$ & $\begin{array}{l}\text { LETTE } \\
\text { R 3 } \\
\text { Mode of } \\
\text { response } \\
\text { to } \\
\text { sensing }\end{array}$ & $\begin{array}{c}\text { LETTER } 4 \\
\text { Programma } \\
\text { bility } \\
\text { Rate } \\
\text { modulation }\end{array}$ & $\begin{array}{c}\text { LETTE } \\
\text { R 5 } \\
\text { Multisite } \\
\text { pacing }\end{array}$ \\
\hline $\mathbf{O}=$ None & $\mathbf{O}=$ None & $\mathbf{O}=$ None & & $\mathbf{O}=$ None \\
\hline $\mathbf{A}=$ Atrium & $\mathbf{A}=$ Atrium & $\begin{array}{c}\mathbf{T}=\text { Trigge } \\
\text { red }\end{array}$ & & $\begin{array}{c}\mathbf{A}=\text { Atriu } \\
\mathrm{m}\end{array}$ \\
\hline $\begin{array}{c}\mathbf{V}=\text { Ventri } \\
\text { cle }\end{array}$ & $\begin{array}{c}\mathbf{V}=\text { Ventri } \\
\text { cle }\end{array}$ & $\begin{array}{c}\mathbf{I}=\text { Inhibit } \\
\text { ed }\end{array}$ & $\begin{array}{c}\mathbf{R}=\text { Rate } \\
\text { modulation }\end{array}$ & $\begin{array}{c}\mathbf{V}=\mathrm{Ventr} \\
\text { icle }\end{array}$ \\
\hline $\begin{array}{c}\mathbf{D}=\text { Dual }( \\
\mathrm{A}+\mathrm{V})\end{array}$ & $\begin{array}{c}\mathbf{D}=\text { Dual }( \\
\mathrm{A}+\mathrm{V})\end{array}$ & $\begin{array}{c}\text { D=Dual }( \\
\mathrm{T}+\mathrm{I})\end{array}$ & & $\mathbf{D}=$ Dual \\
\hline
\end{tabular}

There are a variety of indicatons for pacemakers. Some of the important indications include: sick sinus syndrome, tachy - brady arrhythmia, symptomatic sinus bradycardia, second / third degree AV block with symptoms, RBBB with hemodynamic symptoms, long QT syndrome, cardiomyopathy, syncope without an ECG diagnosis etc... ${ }^{(2)}$ In our patient pacemaker was implanted for repeated syncopal attacks following an ECG diagnosis of complete heart block. 


\section{Types of Pacing Modes}

1.Asynchronous: (AOO, VOO, and DOO)

It is the simple form of pacemaker which discharges at a preset rate. It can be used safely in cases with no ventricular activity. The problem arises when the pacemaker competes with patient's intrinsic rhythm and creates tachyarrhythmias. Continuous pacing wastes energy and also decreases the half-life of the battery.

2.Single Chamber Atrial Pacing (AAI, AAT)

In this system atrium is paced and the impulse passes down the conducting pathways, thus maintaining atrioventricular synchrony. A single pacing lead with electrode is positioned in the right atrial appendage, which senses the intrinsic $P$ wave and causes inhibition or triggering effect.

3.Single Chamber Ventricular Pacing (VVI, VVT) VVI is the most widely used form of pacing in which ventricle is sensed and paced. It senses the intrinsic $\mathrm{R}$ wave and thus inhibits the pacemaker function. patient with complete heart block with chronic atrial flutter, atrial fibrillation and long ventricular pauses.

.4.Dual Chamber AV Sequential Pacing (DDD, DVI, DDI, and VDD)

Two leads one for the right atrial appendage and other for the right ventricular apex are used. The atrium is stimulated first to contract, then after an adjustable PR interval ventricle is stimulated to contract. The normal AV conduction sequence is preserved in this sequence ${ }^{(4)}$.

\section{Internal Cardiac Defibrillators (ICDs)}

VT and VF accounts for majority of sudden cardiac deaths worldwide. Majority of such sudden cardiac deaths occur in a patient with congestive heart failure, ischemic heart disease or dilated cardiomyopathy $^{(6)}$. Later came the advent of ICDs which respond to a dysrhythmia by delivering an internal electric shock within 15 seconds.

Some of the common indications for ICDs include cardiac arrest resulting from VT / VF not resulting from a transient or reversible cause, spontaneous VT with a structural heart disease, Brugada syndrome, Ischemic or non ischemic dilated cardiomyopathy with $\mathrm{EF}<30 \%$ with NYHA class II or III heart failure symptoms, HOCM etc.. ${ }^{(2)}$

The first ICD was implanted by DR LEVI WATKINS at John Hopkins University for a patient by thoracotomy by means of using epicardial leads with a large pulse generator placed in the abdominal cavity. Earlier the dreaded complications of ICDs were spontaneous ventricular dysrhythmias but novel inventions has surpassed these complications. The latest ICDs terminate about $98 \%$ of arrhythmias and the modern ICDs are incorporated with additional pacemaker technology when defibrillation results in bradycardia or asystole ${ }^{(6)}$.

There is also a role for prophylactic ICD insertion in patients with cardiomyopathy and decreased left ventricular function. Numerous clinical trials have proved a survival benefit in such patients. Similar to a pacemaker an ICD also has a generic code to indicate lead placement and function. The NASPE/BPEG generic defibrillation code (NBD code ) is as follows: ${ }^{(2)}$

\begin{tabular}{|c|c|c|c|}
\hline $\begin{array}{l}\text { LETTER } \\
\text { I } \\
\text { Shock } \\
\text { Chambers }\end{array}$ & $\begin{array}{l}\text { LETTER II } \\
\text { Antitachycar } \\
\text { dia Pacing } \\
\text { Chambers }\end{array}$ & $\begin{array}{l}\text { LETTER III } \\
\text { Tachycardia } \\
\text { Detection }\end{array}$ & $\begin{array}{l}\text { LETTER IV } \\
\text { Antibradycar } \\
\text { dia Pacing } \\
\text { Chambers. }\end{array}$ \\
\hline $\begin{array}{l}\mathrm{O}=\text { None } \\
\mathrm{A}= \\
\text { Atrium } \\
\mathrm{V}=\text { Ventric } \\
\mathrm{le} \\
\mathrm{D}=\text { Dual } \\
(\mathrm{A}+\mathrm{V})\end{array}$ & $\begin{array}{l}\mathrm{O}=\text { None } \\
\mathrm{A}=\text { Atrium } \\
\mathrm{V}=\mathrm{Ventricle} \\
\mathrm{D}=\quad \text { Dual } \\
(\mathrm{A}+\mathrm{V})\end{array}$ & $\begin{array}{l}\mathrm{E}= \\
\text { Electrogram } \\
\mathrm{H}=\text { Hemodyna } \\
\text { mic } \\
\text { (Not yet } \\
\text { available) }\end{array}$ & $\begin{array}{l}\mathrm{O}=\text { none } \\
\mathrm{A}=\text { Atrium } \\
\mathrm{V}=\text { Ventricle } \\
\mathrm{D}=\text { Dual }(\mathrm{A}+\mathrm{V} \\
\text { ) }\end{array}$ \\
\hline
\end{tabular}

\section{Perioperative Anaesthetic Significance}

Assistance from the treating cardiologist and the CIED company person should be obtained for assessing the pacemaker function. The type of pacemaker (fixed rate or demand rate), time since it was implanted, pacemaker rate at the time of implantation and half-life of the device battery can be taken from the manufacturer's book kept with the patient. $10 \%$ decrease in rate from the time of implantation indicates power source depletion. Appropriate reprogramming of the pacemaker is more important. It is better to reprogramme the pacemaker from VVI mode to VOO mode the asynchronous mode ${ }^{(5)}$. We did the same thing and 
fixed the rate at 60 beats per minute considering her age and comorbidities.

Detailed history regarding timing of pacemaker is very essential. On insertion of the pacemaker for first two weeks there will be increase in pacing threshold roughly 10 times the acute level. Then it decreases gradually and returns to the initial level in $80 \%$ of patients. This problem is less in steroid eluting leads which has to be enquired from the company person. In our patient the pacemaker was inserted 10 years ago.

Maintaining normokalemia perioperatively is a pivotal part of Anaesthetic management. Its equilibrium across the cell membrane determines the RMP (Resting membrane potential). In hyperkalemia the capture will be more easier, similarly in patients with hypokalemia the capture will be a bit difficult. In our patient normokalemia was maintained throughout the perioperative period. Hypoxia, acidosis and alkalosis must be avoided as it causes increase in pacing threshold.

With respect to the Anaesthesia drugs it has been found that no drug has direct effect on pacing threshold $^{(4)}$. Drugs like succinylcholine, ketamine and etomidate must be avoided as they cause myoclonic movements ${ }^{(3)}$. Hence our patient was induced with Injection propofol and vecuronium bromide. Postoperative shivering has to be avoided as it causes dislodgement of pacemaker leads ${ }^{(3)}$.

\section{Conclusion}

A patient with a CIED in situ is always a special concern for the Anaesthesiologist. Proper history taking, pre anaesthetic stabilization of the patient, Inspection of patient's device information card, coordinating with the company person and cardiologist, anticipation of complications will help in safe management of the patient and the device. Last but not least, facility for transcutaneous pacing and transvenous pacing must be always ready in the OR

\section{References}

1. Ketaki Patwardhan, Josna Kamble, Aniruddha Nirkhi. "Anesthetic Management of Elderly
Patient with Permanent Pacemaker for Excision of Suspected Adrenal Mass - A Challenging Case". Journal of Evolution of Medical and Dental Sciences 2014; Vol. 3, Issue 04, January 27; Page: 976-981, DOI: 10.14260/jemds/2014/1935

2. Pacemakers \& Implantable CardioverterDefibrillators (Icds) - Part 1 Anaesthesia Tutorial Of The Week 299 25th November 2013 Dr. Md. Rajib Pervez, Trainee in Anaesthesiology, Bangladesh Medical College Hospital (BMCH), Bangladesh.

3. Perioperative anaesthetic management of patients with cardiac pacemakers in non-cardiac surgery- A Case Report Dr Priti R Sanghavi, Dr Kinna G Shah, Dr Nikunjal J Patel, Dr Gaurav Sharma, Dr Surbhi Goyal, Dr Bipin M Patel Resident, Assistant Professor,Professor, Professor \& HOD Dept. of Anaesthesiology, Department of Anaesthesiology, Gujarat Cancer and Research Institute, Ahmedabad

4. Anaesthetic Management of Patients with Cardiac Pacemakers and Defibrillators for Noncardiac Surgery Shivani Rastogi, MD, Sanjay Goel, MD, Deepak K Tempe, MD, Sanjula Virmani, DA, DNB Department of Anaesthesiology and Intensive Care, GB Pant Hospital, New Delhi

5. Pacemakers and de®brillators: anaesthetic implications T. V. Salukhe1*, D. Dob2 and R. Sutton 1 National Heart Lung Institute, Royal Brompton Hospital, Sydney Street, London SW3 6NP, UK. Chelsea and Westminster Hospital, London, UK*Corresponding author. Pacing and ICD Dept. E-mail: t.salukhe@imperial.ac.uk Br J Anaesth 2004; 93: $95 \pm 104$

6. Perioperative management of patients with cardiac implantable electronic devices M. E. Stone1*, B. Salter1 and A. Fischer2rg 1 Department of Anaesthesiology and 2 Department of Medicine, Mount Sinai School of Medicine, New York, NY, USA. 